\title{
El meningioma en edad pediátrica. Revisión de 10 casos
}

\author{
P. Teixidor; A. Guillén*; O. Cruz** y J.M. Costa*
}

Servicio de Neurocirugía. Hospital de Bellvitge. Barcelona. Servicios de Neurocirugía* y Oncología**. Hospital Sant Juan de Déu. Barcelona.

\section{Resumen}

La presencia de meningiomas en la edad pediátrica es relativamente rara, representan menos del $5 \%$ de todos los tumores del sistema nervioso central en este estadio de la vida.

Hemos realizado un estudio retrospectivo de 10 casos de meningiomas en pacientes pediátricos analizando los factores epidemiológicos, clínicos y radiológicos, así como el pronóstico a largo plazo de nuestros pacientes. Los resultados se comparan con aquéllos reportados en la literatura.

PALABRAS CLAVE: Meningioma. Neoplasia. Sistema nervioso central. Pediatría.

Intracranial meningiomas in children: report of $\mathbf{1 0}$ cases

\section{Summary}

The occurrence of meningiomas in children is rare, accounting for less than $5 \%$ of tumors of the central nervous system in childhood. This is a retrospective study of 10 patients with CNS meningiomas. The goal of this study was to determine the epidemiology, clinical and radiological features, and long-term outcome of meningiomas The results ware compared with those reported in the literature.

KEY WORDS: Meningiomas. Neoplasia. Central nervous system. Pediatric.

\section{Introducción}

El meningioma es un tumor poco habitual en la edad pediátrica aunque no es sólo su baja frecuencia la única desigualdad de esta neoplasia respecto a los adultos.

Recibido: 12-07-07. Aceptado: 10-12-07
Presentamos una serie propia de 10 niños diagnosticados e intervenidos de meningioma intracraneal, junto con una revisión de la literatura, con el objetivo de determinar las diferencias (epidemiológicas, clínicas, radiológicas...) de este grupo tumoral en distintas etapas de la vida.

\section{Material y métodos}

La serie retrospectiva fue extraída de la base de datos del servicio de Neurocirugía del Hospital Sant Joan de Déu de Barcelona, entre todos los pacientes intervenidos de tumor cerebral entre 1981 y 2003 . Se extrajeron de la historia clínica los datos correspondientes a la epidemiología (edad al diagnóstico, sexo y antecedentes patológicos de interés), clínica en el momento del diagnóstico (presencia de crisis comiciales, focalidad neurológica, alteraciones cognitivas o clínica de hipertensión intracraneal), y neuroradiología (características con y sin contraste en TAC y RM, localización anatómica, inserción de la lesión, tamaño, presencia de edema, desplazamiento de la línea media, y área de elocuencia). Respecto a los datos quirúrgicos se valoró el grado de resección (mediante TAC y/o RM postquirúrgica), y las complicaciones neurológicas, regionales, y sistémicas secundarias a la intervención. Mediante los controles radiológicos y por los datos clínicos anotados en consultas externas se valoró la evolución de los mismos.

\section{Resultados}

\section{Epidemiología}

La serie constaba de 10 pacientes, de edades entre un mes y los 13 años (media de edad de 8 años DS +/- 5.2). Cinco de ellos eran varones y cinco mujeres. Del total de la serie, tan sólo dos tenían antecedentes de interés para este tipo de patología, dos de ellos estaban afectos de neurofibromatosis tipo I y otro fue irradiado a nivel cranial, 6 años antes, por restos tumorales tras la resección a los dos años de un meduloblastoma en fosa posterior.

El seguimiento medio fue de 23.7 meses ( entre 4 y 60 meses). De una paciente, tras 18 meses de seguimiento, no se obtuvo más información. 


\section{Clínica}

El debut de la enfermedad fue en forma de crisis comiciales en 6 pacientes (6/10), con focalidad neurológica en dos de ellos, alteraciones cognitivas en dos y con signos de hipertensión intracraneal en otros dos pacientes. El reconocimiento del paciente evidenció la presencia de una tumoración craneal en tres niños.

\section{Radiología}

De los diez casos, nueve se localizaban a nivel supratentorial, siendo cuatro de ellos frontales. Los diámetros máximos de las lesiones tenían una media de $41.9 \mathrm{~mm}$ (DS+/- 21.7mm), y $35.1 \mathrm{~mm}$ (DS+/- 16.2mm). El tumor se presentaba isointenso en la RM del diagnóstico en cinco casos, y de forma quística al TAC y RM en 5 de los 10 . Se encontraba la presencia de edema en 3 pacientes, de ellos el edema era menor al volumen tumoral y en un caso mayor. La línea media se desplazaba más de un centímetro en tres casos. El meningioma se hallaba en región elocuente en 6 pacientes.

\section{Cirugía}

La resección (comprobada mediante TAC o RM posquirúrgica) fue completa en 6 casos, incompleta en cuatro casos, de los cuales en 2 se pudo realizar una casi completa extirpación del tumor. Como complicaciones neurológicas destacó un caso con focalidad motora recuperada a los dos meses de la intervención y un caso con disnomia leve persistente. De las complicaciones regionales, un caso presentó fístula de líquido cefalorraquídeo por herida quirúrgica (caso 6) con la posterior infección de la herida, sin infección profunda, pero complicándose con sepsis; esta fue la única complicación sistémica hallada.

\section{Anatomía patológica}

De los diez pacientes intervenidos, tres de ellos eran meningiomas fibrosos, uno meningotelial, uno transicional, dos angiomatosos y uno atípico, en dos casos únicamente se estableció el diagnóstico.

\section{Seguimiento}

Se confirmó recidiva y/o progresión tumoral en 4 pacientes (50\%). Precisaron reintervención 3 casos, y/o radioterapia otros tres. El diagnóstico de la recidiva se realizó como media a los 14 meses (entre los 9 y los 24 meses).

\section{Discusión}

\section{Epidemiología}

La incidencia de meningioma en las edades pediátricas oscilaba entre el $1.1 \%$ al $4.5 \%$ de todos los tumores pediátricos intervenidos ${ }^{1,4,5,8,10,11}$; mientras que del global de los meningiomas intervenidos representaban tan sólo entre el $1.08 \%$ al $3.3 \%{ }^{1,5,10}$.

Las series revisadas fueron series de pacientes en edades pediátricas pero el rango de edades variaba, puesto que en función del centro se consideraron hasta los 15 ó hasta los 20 años, hecho que implicaba un aumento en la

Tabla I

Datos biológicos y clínicos en 10 pacientes pediátricos con meningioma intracraneal

\begin{tabular}{|c|c|c|c|c|c|c|c|c|}
\hline $\mathbf{N}^{\mathbf{o}}$ & Sexo & $\begin{array}{l}\text { Edad } \\
\text { años }\end{array}$ & $\mathbf{A P}$ & Epilepsia & $\begin{array}{l}\text { Focalidad } \\
\text { inicio }\end{array}$ & $\begin{array}{l}\text { Deterioro } \\
\text { cognitivo }\end{array}$ & HIC & $\begin{array}{c}\text { Tumoración } \\
\text { cranial }\end{array}$ \\
\hline 1 & $\lambda$ & 12 & $\begin{array}{c}\mathrm{Si} \\
\text { (neurofib) }\end{array}$ & No & No & No & $\mathrm{Si}$ & $\mathrm{Si}$ \\
\hline 2 & q & 12 & No & $\mathrm{Si}$ & No & No & No & No \\
\hline 3 & 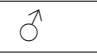 & 10,5 & No & $\mathrm{Si}$ & No & No & No & No \\
\hline 4 & $q$ & 3 & No & No & $\mathrm{Si}$ & $\mathrm{Si}$ & No & No \\
\hline 5 & $q$ & 13 & No & $\mathrm{Si}$ & $\mathrm{Si}$ & No & $\mathrm{Si}$ & No \\
\hline 6 & q & 0,2 & No & No & No & No & No & $\mathrm{Si}$ \\
\hline 7 & $\pi$ & 12 & No & $\mathrm{Si}$ & No & No & No & No \\
\hline 8 & $\pi$ & 8 & $\begin{array}{c}\mathrm{Si} \\
\text { (irradiación) }\end{array}$ & $\mathrm{Si}$ & No & $\mathrm{Si}$ & No & No \\
\hline 9 & $\pi$ & 11 & No & $\mathrm{Si}$ & No & No & No & No \\
\hline 10 & q & 0 & No & No & No & No & No & $\mathrm{Si}$ \\
\hline
\end{tabular}


Tabla 2

Datos radiológicos de la serie

\begin{tabular}{|c|c|l|c|c|c|c|}
\hline $\mathbf{N}^{\mathbf{0}}$ & Diame 1 & Diame 2 & Elocuencia & Inserción dural & Edema & Desplaz. línea media \\
\hline 1 & 80 & 28 & Paraelocuente & Convexidad & No & No \\
\hline 2 & 30 & 30 & Elocuente & Ala menor esfenoides & No & Si $<0,5$ \\
\hline 3 & 45 & 40 & Elocuente & Tentorio & Si menor al $>1 \mathrm{~cm}$ \\
\hline 4 & 66 & 60 & Elocuente & Ala menor esfenoides & Solumen tumoral & Si menor al $0,5-1$ \\
\hline 5 & 40 & 40 & Elocuente & Hoz & No & Si mayor al \\
\hline 6 & 33 & 28 & Elocuente & Fosa posterior & No & Nolumen tumoral \\
\hline 7 & 20 & 20 & Elocuente & Hoz & No & Si \\
\hline 8 & 20 & 20 & No elocuente & Convexidad & No & No \\
\hline 10 & 65 & 65 & Paraelocuente & Ala menor esfenoides & Ala menor esfenoides & No \\
\hline
\end{tabular}

Tabla 3

Datos respecto a la cirugía, anatomía patológica y seguimientos

\begin{tabular}{|c|c|c|c|c|c|c|}
\hline Caso & Año & Grado resección & Anatomía patológica & Recidiva & $\begin{array}{l}\text { Tratamiento } \\
\text { recidiva }\end{array}$ & $\begin{array}{l}\text { Momento } \\
\text { recidiva }\end{array}$ \\
\hline 1 & 2001 & Resección completa & Meningioma fibrós & Perdido & & \\
\hline 2 & 1994 & Resección completa & $\begin{array}{c}\text { Meningioma mixte } \\
\text { anaplásic }\end{array}$ & No & & \\
\hline 3 & 1986 & Resección parcial & Meningioma & Recidiva & IQ+RAD & $24 \mathrm{M}$ \\
\hline 4 & 2003 & Resección completa & Meningioma fibrós & No & & \\
\hline 5 & 2000 & Resección parcial & $\begin{array}{l}\text { Meningioma menin- } \\
\text { gotelial sincitial }\end{array}$ & Recidiva & $\mathrm{IQ}+\mathrm{RAD}$ & $9 \mathrm{M}$ \\
\hline 6 & 2002 & Resección completa & Meningioma fibrós & No & & \\
\hline 7 & 2003 & Resección completa & Meningioma angiomatós & No & & \\
\hline 8 & 1981 & Resección completa & Meningioma transicional & No & & \\
\hline 9 & 1991 & Resección parcial máxima & Meningioma & Recidiva & RAD & $12 \mathrm{M}$ \\
\hline 10 & 1991 & Resección parcial máxima & Meningioma angioblástic & Recidiva & IQ & $12 \mathrm{M}$ \\
\hline
\end{tabular}

incidencia en estas últimas ${ }^{3}$.

Se contabilizaron, junto con nuestra serie un total de 76 varones frente a las 68 mujeres entre todas las series $^{1,4,5,6,8,10,11}$, resultando una razón (varones/mujeres) de 1,11. Resultó mínima la mayor incidencia en estas edades en los varones, contrastando notablemente con la clara afectación en las mujeres en las edades más adultas.

Las edades medias en la bibliografía oscilaban entre los

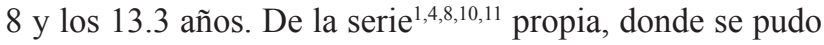

objetivar edad y sexo destacamos un predominio en varones en edades más tempranas con tendencia a equilibrarse entre los 6 y los 10 años, para finalmente aumentar la incidencia en el sexo femenino. (Tabla 4).

\section{Clínica}

Son pocas las series que recogen la duración de los síntomas antes de establecerse el diagnóstico. En dos de ellas ${ }^{6,11}$ la media osciló entre los 4.5 meses y los 12 meses respectivamente. Respecto a la clínica, la cefalea (como manifes- 
El meningioma en edad pediátrica. Revisión de 10 casos

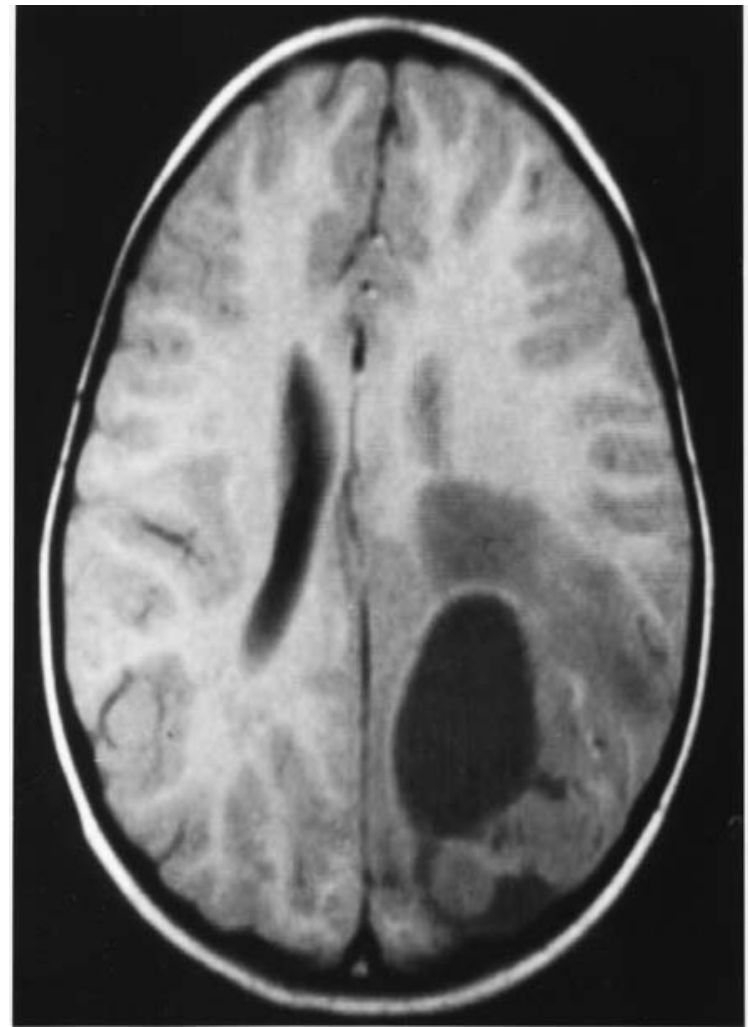

RMN sin contraste axial mostrando lesión parietooccipital izquierda (caso número 5).

tación de hipertensión intracraneal) se detectó en 56 de 122 pacientes $^{1,4,5,6,10,11}$, (propia), resultando pues el síntoma más habitual al diagnóstico ( $45 \%$ de los casos). Seguidamente, la focalidad neurológica (paresia, parestesias, alteración visual...) fue el segundo más frecuente en la clínica (31\%, $38 / 122$ ), siguiéndose por las crisis comiciales en $29 / 122$ $(23 \%)$. Se evidenció una tumoración craneal a la exploración en un total de 9 pacientes $(7,3 \%)$.

Observamos pues que la presentación clínica de los meningiomas en edades pediátricas fue superponible a la misma en las edades adultas con pequeñas distinciones.
Neurocirugía

2008; 19: 434-439

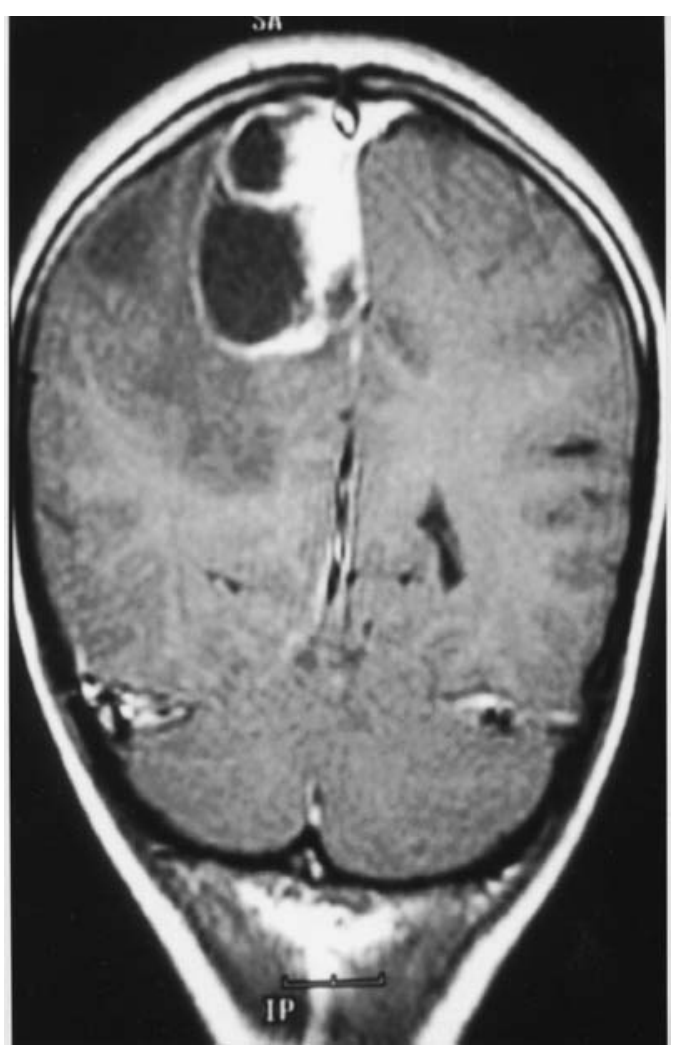

RMN coronal con contraste de una lesión parasagital con invasión del seno longitudinal superior (caso número 7).

\section{Localización y neuroimagen}

Para determinar las localizaciones más habituales de este tipo de tumor observamos que en las distintas series no se utilizaron los mismos criterios de clasificación. Las series que diferenciaron una situación supratentorial respecto a la infratentorial ${ }^{4,5,8}$ obtuvimos un total de $60 / 74$ (81\%) supratentoriales respecto a los 12 infratentoriales $14 / 74$ (18.9\%). De las series que mencionaron la implantación intraventricular ${ }^{4,5,6,11}$, se aislaron un total de 11 casos entre 79 (13.9\%). Destacamos a su vez, emplazamientos

Tabla 4

Edad y sexo de los pacientes en las series consultadas

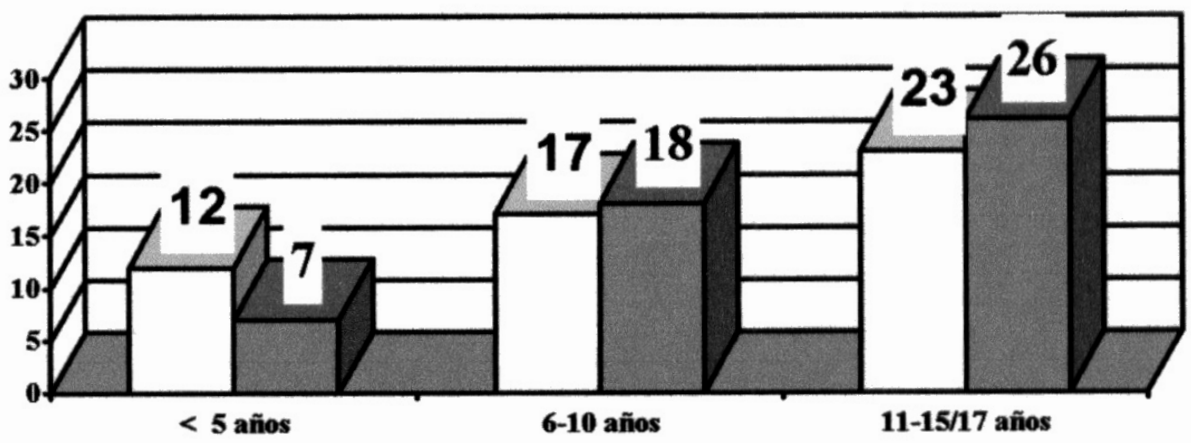

口varones 口hembras 
atípicos como tres casos en la fisura silviana, 1 en cisterna ambiens, un tumor en seno esfenoetmoidal...

El tamaño de este tipo de tumor en las series analizadas quedó registrado en muy pocas series de ellas, siendo mayor de $5 \mathrm{~cm}$ en el $50 \%{ }^{1}$, en el $55 \%{ }^{4}$ y como media de $5.7 \mathrm{~cm}(3-9 \mathrm{~cm})$ en la serie $\mathrm{de}^{11}$. Respecto a nuestra serie particular, 3 de ellos tuvieron un diámetro máximo superior a $5 \mathrm{~cm}$, mientras que la media del diámetro máximo fue de $4.19 \mathrm{~cm}$. Estos datos, sugieren que los meningiomas en las edades pediátricas alcanzan tamaños considerables.

Son muy pocas las series que describen las características de presentación en el TAC y/o RM. Destacamos que en la serie de So-Hyang ${ }^{11}$, donde queda ampliamente detallados los hallazgos en la RM, en 7 de 11 casos se presentaron con formaciones quísticas intratumorales con la consecuente captación heterogénea de contraste. La serie propia constaba de 4 casos con formaciones quísticas por neuroimagen. Por contra existen series donde no hallaron ningún caso con formación quística ${ }^{1,4}$. De nuestra serie, solo 5 casos de los diez presentaban por imagen una presentación característica de meningioma. Las presentaciones atípicas de los meningiomas en edades pediátricas parece que son más frecuentes que en los adultos, aunque son pocas las series donde quede reflejado.

\section{Anatomía patológica}

En cuanto a la anatomía patológica de los meningiomas pediátricos de las series revisadas junto a la propia ${ }^{1,4}$, $5,6,10,11$, la más habitual al igual que en los adultos fue los meningoteliales $47 / 122$ (38,5\%), seguido de los transicionales 28/122 (22.9\%), los fibrosos 17/122 (13.9\%) у psamomatosos $9 / 122(7.3 \%)$.

\section{Cirugía}

Con los datos de nuestra serie y las demás series valoradas $^{1,4,5,6,10,11}$, se obtuvo una resección completa en un total de 93 casos $/ 122(76,2 \%)$. Entre estos, presentaron recurrencia 13 casos $(13.9 \%)$ tras una media de seguimiento entre los 2.8 años y los 18 años según la serie.

Comparando estos datos con los correspondientes a las edades adultas, observamos que el grado de recurrencia en edades pediátricas es superior al de los adultos (según Bárbaro el $96 \%$ de los meningiomas extirpados completamente en edad adulta, durante un periodo de seguimiento entre los 5 y los 15 años, quedan libres de recurrencia).

La mayor parte de las resecciones incompletas son justificadas por los autores, por la localización del tumor (seno cavernoso, tórcula, clivus.... $)^{1,4}$.

Respecto a las recidivas en las resecciones subtotales, los datos hallados en la literatura son muy dispares y poco concluyentes. Algunas series no mencionan la evolución de estos pacientes, en otros el tiempo de seguimiento es reducido para el comportamiento de este tipo de tumor (durante
5 años tres de los tres pacientes con resecciones subtotales no recidivan ${ }^{4}$ ). De nuestra serie, los cuatro pacientes con exéresis parcial, los cuatro recidivaron a los 14 meses de media recibiendo reintervención y/o radioterapia como tratamiento.

\section{Conclusiones}

El meningioma en edades pediátricas presenta una incidencia mucho menor que en las edades más avanzadas. Este factor hace que la experiencia quede reducida respecto estas edades. Es característica la mayor afectación de los varones en las edades más tempranas para invertirse a partir de los 10 años a favor de las mujeres.

La presentación clínica a lo largo de los años no parece presentar grandes variaciones.

Algunas localizaciones (intraventriculares), poco habituales en adultos, son más frecuentes en edades infantiles.

El tamaño alcanzado en las edades pediátricas en el momento del diagnóstico llega a ser considerable.

La presentación por imagen es más anómala que en las edades más avanzadas.

La anatomía patológica no varía con los años.

El comportamiento tumoral sugiere ser más agresivo en las edades más cortas.

\section{Bibliografía}

1. Amirjamshidi, A., Mehrzin, M., Abbassioun, K.: Meningiomas of the central nervous system occurring below the age of 17: report of 24 cases not associated with neurofibromatosis and review of literature. Child's Nery Syst 2000; 16: 406-416.

2. Bárbaro, N.M., Gutin, P.H., Wilson, C.B., et al.: Radiation therapy in the treatment of partially resected meningiomas. Neurosurgery $1987 ; 20: 525-528$.

3. Di Rocco, C., Di Rienzo, A.: Meningiomas in childhood. Crit Rev Neurosurg 1999, 9: 180-188.

4. Edingler, P., Lena, G., Sarioglu, A.G., et al.: Intracranial meningiomasin children: review of 29 cases. Surg Neurol 1998; 49: 136-141.

5. Edwards, M., Davis, R., et al. Germano I.: Intracranial meningiomas of the first two decades of life. J Neruosurg 1994; 80: 447- 453.

6. Mallucci, C.L., Parkes, S.E., Barber, P., et al.: Paediatric meningeal tumours. Child's Nery Syst 1996; 12: 582589.

7. Neurosurgical Surgery. Youmans. New York, Saunders, 2004. Chapter 62 ( 1099-1139). Winn. Meningiomas.

8. Rochart, P., Hjorth, H., Gjerris, F.: Long-term follow up of children with meningiomas in Denmark K: 1935-1984. J Neurosurg (Pediatrics 2) 2004; 100: 179-182.

9. Sakaki, S., Nakagawa, K., Kimura, H., et al.: Intra- 
cranial meninigomas in infancy. Surg Neurol 1987; 28: 5157.

10. Sheikh, B.Y., Sigueira, E., Dayel, F.: Meningioma in children: a report of nine cases and review of the literature. Surg Neurol 1996; 45: 328-335.

11. So-Hyang, I., Kyu- Chang, W., Seung-Ki, K., et al.: Childhood meningioma: unusual location, atypical radiological findings and favorable treatment outcome. Child's Nery
Syst 2001; 17: 656-662.

Teixidor, P.; Guillén, A.; Cruz, O.; Costa, J.M.: El meningioma en edad pediátrica. Revisión de 10 casos. Neurocirugía 2008; 19: 434-439.

Correspondencia postal: Antonio Guillén Quesada. Hospital Sant Joan de Deu. Paseo Sant Joan de Deu, 2. 08950 Barcelona. 